\title{
Performance of the TPC with micro pixel chamber readout: Micro-TPC
}

\section{$\operatorname{AUTHOR}(\mathrm{S})$ :}

Miuchi, K; Kubo, H; Nagayoshi, T; Ochi, A; Orito, R; Takada, A; Tanimori, T; Ueno, M

\section{CITATION:}

Miuchi, K ... [et al]. Performance of the TPC with micro pixel chamber readout: Micro-TPC. IEEE TRANSACTIONS ON NUCLEAR SCIENCE 2003, 50(4): 825-830

\section{ISSUE DATE:}

2003-08

URL:

http://hdl.handle.net/2433/49930

\section{RIGHT:}

(c)2003 IEEE. Personal use of this material is permitted. However, permission to reprint/republish this material for advertising or promotional purposes or for creating new collective works for resale or redistribution to servers or lists, or to reuse any copyrighted component of this work in other works must be obtained from the IEEE. 


\title{
Performance of the TPC With Micro Pixel Chamber Readout: Micro-TPC
}

\author{
Kentaro Miuchi, Hidetoshi Kubo, Tsutomu Nagayoshi, Atsuhiko Ochi, Reiko Orito, Atsushi Takada, Toru Tanimori, \\ and Masaru Ueno
}

\begin{abstract}
Micro-TPC, a time projection chamber (TPC) with micro pixel chamber ( $\mu$-PIC) readout, was developed for the detection of the three-dimensional $(3-D)$ fine $(\sim$ submillimeter $)$ tracks of charged particles. We developed a two-dimensional position sensitive gaseous detector or the $\mu$-PIC, with the detection area of $10 \times 10 \mathrm{~cm}^{2}$ and 65536 anode electrodes of $400 \mu \mathrm{m}$ pitch. We achieved the gas gain of more than $10^{4}$ without any other multipliers. With the pipeline readout system specially developed for the $\mu$-PIC, we detected X-rays at the maximum rate of $7.7 \mathrm{MHz}$. We developed a micro-TPC with the $\mu$-PIC and 3-D tracks of electrons were detected with the micro-TPC. We also developed a prototype of the MeV gamma-ray imaging detector which is a hybrid of the micro-TPC and NaI (TI) scintillator so that we showed that this is a promising method for the $\mathrm{MeV}$ gamma-ray imaging.
\end{abstract}

Index Terms-Gamma-ray imaging, gaseous detector, micropattern detector, time projection chamber.

\section{INTRODUCTION}

$\mathbf{R}$ EALIZATION of a micro-TPC, a time projection chamber (TPC), which can detect three-dimensional (3-D) fine tracks of charged particles, has been wanted for years. We developed the $\mu$-PIC, a two-dimensional (2-D) fine position detector, and a fast readout electronics in order to realize such an "electric cloud chamber." In this paper, the performance of the micro-TPC, together with one example of its applications, is described.

\section{II. $\mu$-PIC DETECTOR}

The micro pixel chamber or the $\mu$-PIC, is a pixel type gaseous 2-D imaging detector, which takes over the outstanding properties of the MicroStrip Gas Chamber (MSGC [1], [2]) such as the good position resolution and operating capacities under high flux irradiation. The $\mu$-PIC is manufactured by the print circuit board (PCB) technology in contrast to an older concept pixel type detector, the microdot chamber [3], which is made in the MOS technology. With the PCB technology, large area detectors can be made cheaply, which is an important feature for developing various kinds of applications. As a consequence of the

Manuscript received December 1, 2002; revised April 2, 2003. This work was supported by a Grant-in-Aid in Scientific Research of the Japan Ministry of Education, Culture, Science, Sports, and Technology and "Ground Research Announcement for Space Utilization" promoted by the Japan Space Forum.

K. Miuchi, H. Kubo, T. Nagayoshi, R. Orito, A. Takada, T. Tanimori, and M.Ueno are with the Department of Physics, Graduate School of Science, Kyoto University, Sakyo-ku, Kyoto 606-8502, Japan (e-mail: miuchi@cr.scphys. kyoto-u.ac.jp).

A. Ochi is with the Department of Physics, Graduate School of Science and Technology, Kobe University, Kobe 657-8501, Japan.

Digital Object Identifier 10.1109/TNS.2003.814564

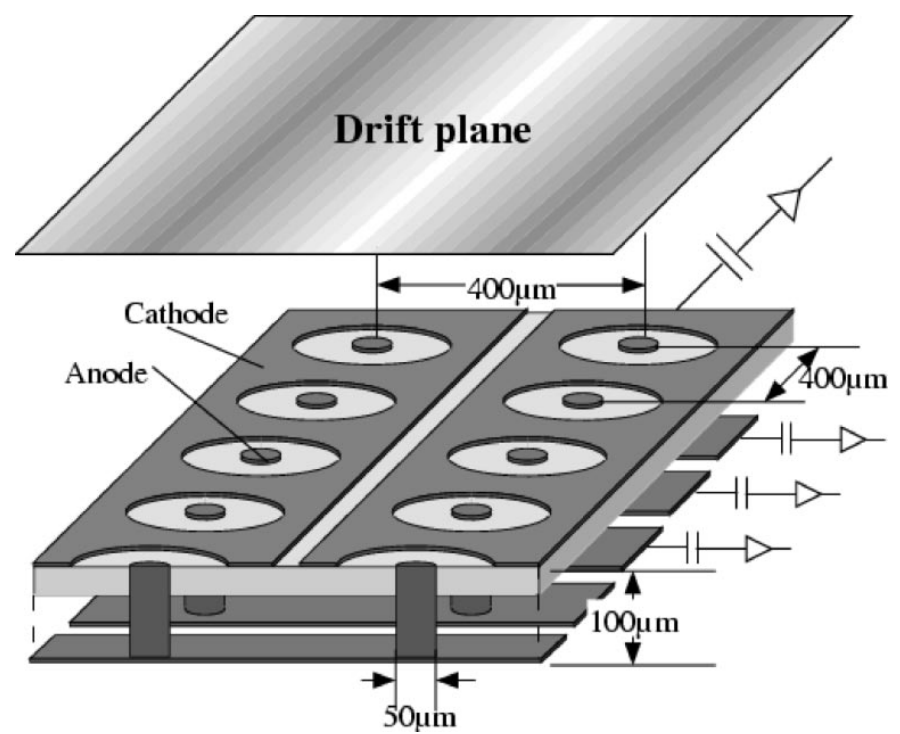

Fig. 1. Schematic structure of the $\mu$-PIC.

geometrical properties, discharge problems are less disturbing with $\mu$-PIC [4]-[6], so that stable operations at high gas gain can be realized.

The schematic structure of the $\mu$-PIC is shown in Fig. 1 . The $\mu$-PIC is a double-sided PCB with a $100 \mu \mathrm{m}$-thick polyimide substrate. Two hundred fixty six anode strips are formed on one side of the $\mu$-PIC, while 256 cathode strips are orthogonally placed on the other side. Both anode and cathode strips are placed with a pitch of $400 \mu \mathrm{m}$. Cathode strips have holes of $200 \mu \mathrm{m}$ diameter and anode electrodes of 50-70 $\mu \mathrm{m}$ diameter are formed on the anode strips at the center of each cathode hole. The signals from anode strips and cathode strips are of the same size, in contrast to the MSGCs, whose pulse heights from back strips are $20 \%-30 \%$ of those from anode strips [2]. We developed the $\mu$-PIC of $10 \times 10 \mathrm{~cm}^{2}$ detection area with $256 \times 256$ anode electrodes. The $\mu$-PIC is mounted on the mother board of $30 \times 30 \mathrm{~cm}^{2}$ area by the bonding technique.

We irradiated a noncollimated ${ }^{55} \mathrm{Fe}$ radioactive source and measured the output charge from $32 \times 256$ pixels so that we calculated the gas gain. The gas mixture of argon $80 \%$ and ethane $20 \%$ at 1 atm was flowed for the measurement. The gas gain of the $\mu$-PIC is plotted as a function of the anode voltage in Fig. 2. We achieved a maximum gas gain of $1.5 \times 10^{4}$ and the gas gain for the stable operation of 5000 without any other multipliers. In Fig. 3, the spectrum of the X-rays from the ${ }^{55} \mathrm{Fe}$ radioactive source is shown. The energy resolution is 30\% (FWHM) for the $5.9 \mathrm{keV}$ X-rays. This result is worse than our previous results 


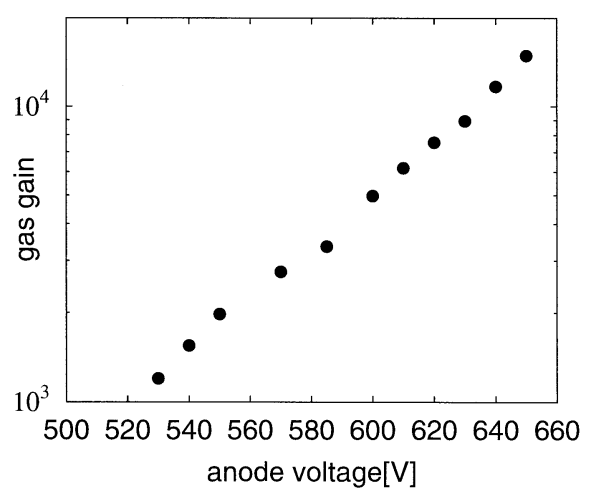

Fig. 2. Gas gains as a function of the anode voltage.

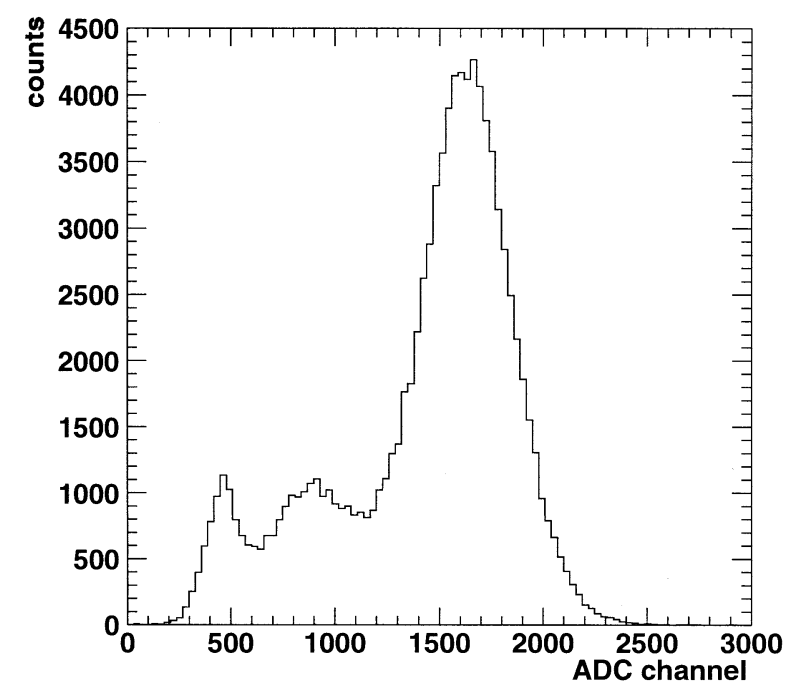

Fig. 3. Obtained spectrum of the $\mathrm{X}$-rays from a ${ }^{55} \mathrm{Fe}$ radioactive source.

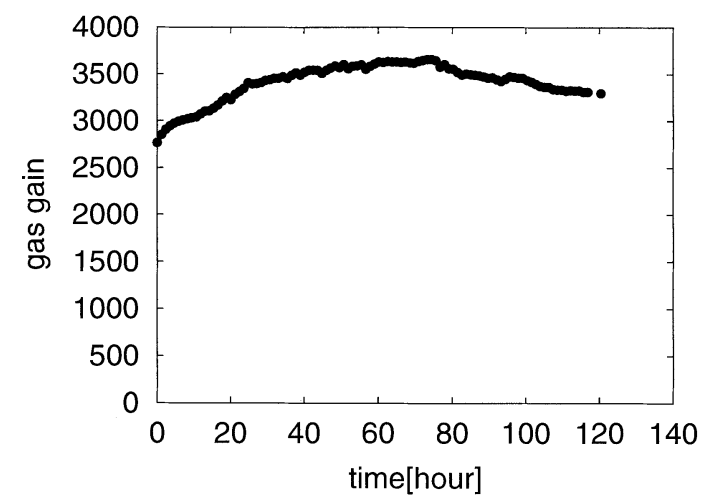

Fig. 4. Gas gains as a function of the time.

$20 \%$ obtained with the trial piece which has $3 \times 3 \mathrm{~cm}^{2}$ detection area [4]. The nonuniformity of the anode electrodes in the large detection area makes the resolution worse. New technologies for forming the uniform electrodes are now sought for so as to achieve higher gas gain and better energy resolution.

Long-term stability is a necessary feature in order to realize the various applications of the $\mu$-PIC. We operated the $\mu$-PIC for $120 \mathrm{~h}$ with the gain of more than 3000 without serious discharge. Gas gain was measured every one hour by the same method described above. The gain variation is shown in Fig. 4 as a func-

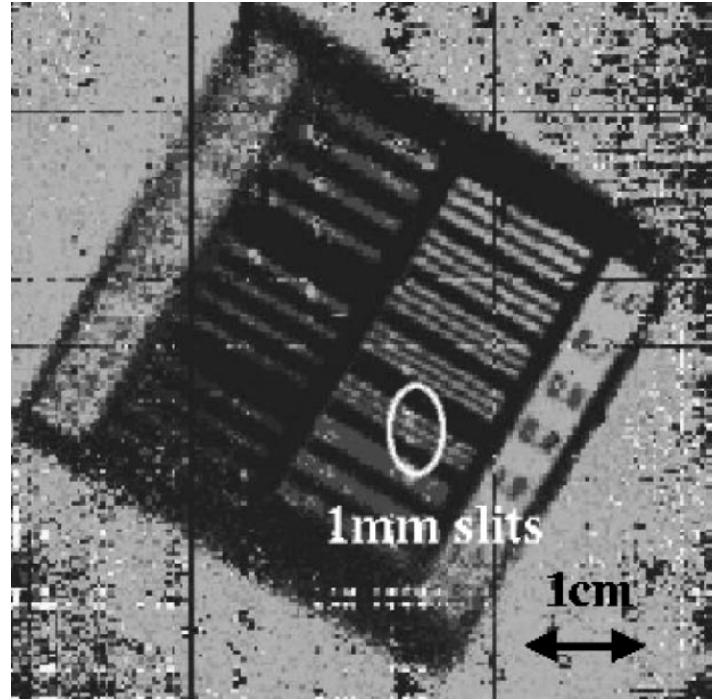

Fig. 5. The X-ray image of the test chart.

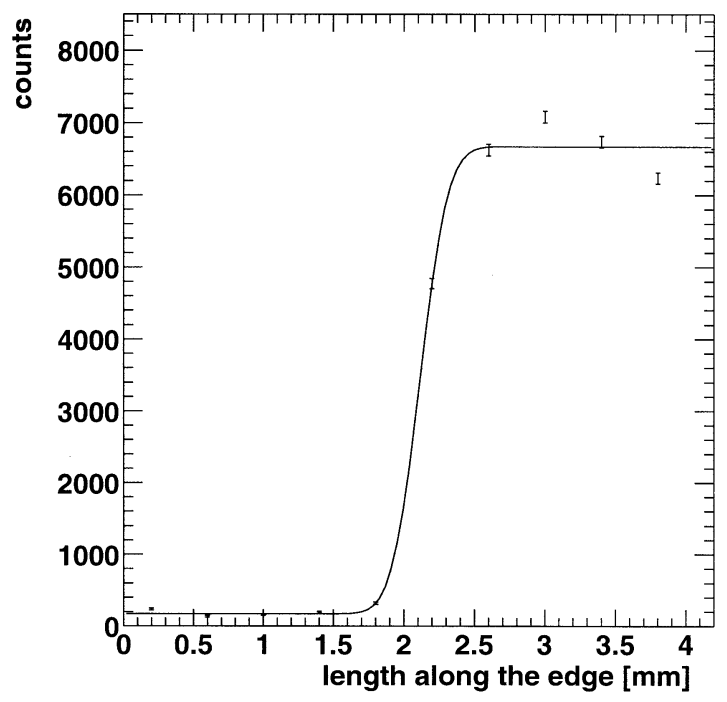

Fig. 6. Projected image of the test chart edge and the best fit function.

tion of the elapsed time since the anode voltage supply. Slight increase of the gain was observed in the first $20 \mathrm{~h}$, which we think is due to the polarization effect of the substrate. The gain was stable within $10 \%$ after $20 \mathrm{~h}$.

We took the X-ray image of the test chart with an X-ray generator (Kevex X-Ray CU028, tungsten target). The acceleration voltage of the X-ray generator was $18 \mathrm{kV}$. Low-energy $\mathrm{X}$-rays were filtered with $50 \mu \mathrm{m}$ thick copper and $1 \mathrm{~mm}$ thick aluminum and the energy of the X-rays was distributed between 14 and $18 \mathrm{keV}$ with the intensity peak at $17 \mathrm{keV}$. We used the gas mixture of xenon $70 \%$ and ethane $30 \%$ at 1 atm. We chose xenon gas in order to achieve clear images. The practical range of the $20 \mathrm{keV}$ electron in argon gas is $3 \mathrm{~mm}$ while that in the xenon gas is $1 \mathrm{~mm}$. The detection depth was set to be $2 \mathrm{~mm}$. The test chart is $5 \times 5 \mathrm{~cm}^{2}$ area and slits of various widths are scribed in the thin lead layer on the plastic plate. In Fig. 5, obtained X-ray image is shown. The slits of $1 \mathrm{~mm}$ width are seen clearly. 


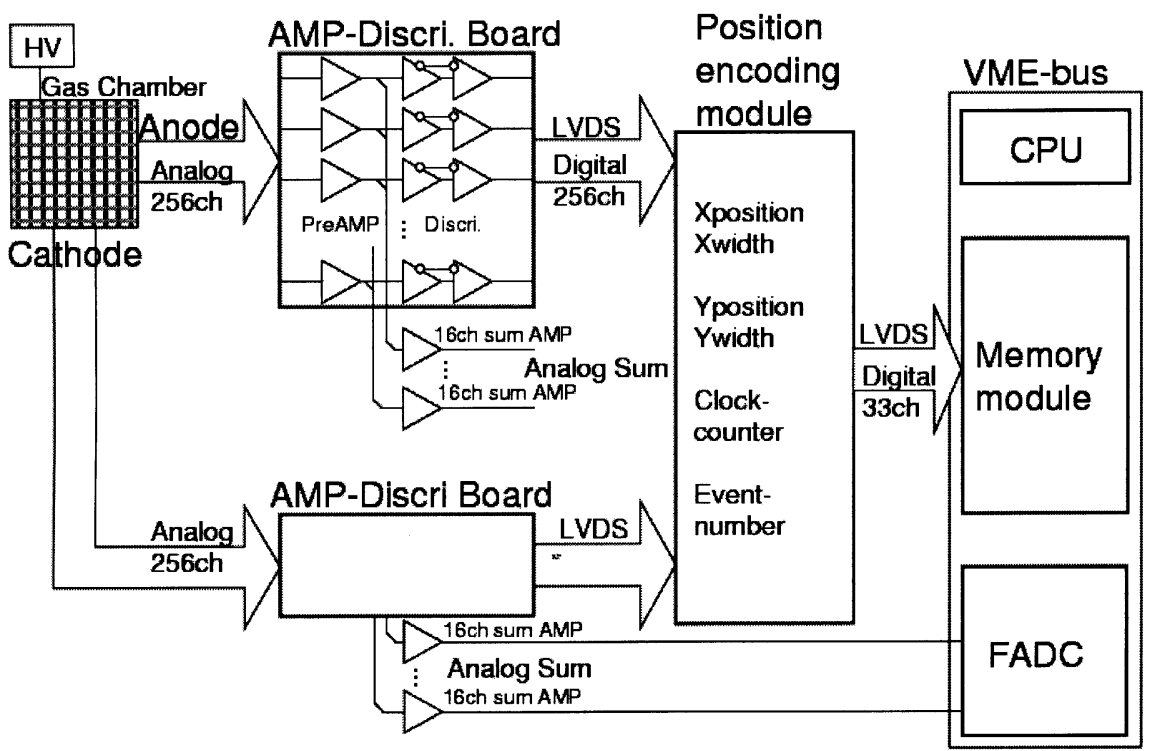

Fig. 7. Block diagram of the readout electronics.

We measured the spatial resolution from the edge image of the test chart. Acceleration voltage of the X-ray generator was set at $10 \mathrm{kV}$ for this measurement in order to suppress the range of the electrons in the xenon gas. The active area was set to be $4 \times 8 \mathrm{~cm}^{2}$ with one of the edges at the center of the active area. The test chart image was projected along one of the edges for $1.3 \mathrm{~cm}$. The projected image was fitted with the function $a_{1}+$ $a_{2} \cdot \operatorname{erf}\left(\left(x-a_{3}\right) / \sqrt{2} a_{4}\right)$, where $\operatorname{erf}(x)=2 / \sqrt{\pi} \cdot \int_{0}^{x} \exp \left(-t^{2}\right) d t$ is the error function and $a_{1}, a_{2}, a_{3}$ and $a_{4}$ are the fitting parameters. The calculated spatial resolution $\left(a_{4}\right)$ was $160 \mu \mathrm{m}$. The histogram of the projected image of the test chart and the best fit function is shown in Fig. 6.

\section{READOUT ELECTRONICS}

A pipeline readout electronics system for the $\mu$ PIC was developed as well as the detector itself [7]. The block diagram of the readout electronics is shown in Fig. 7. Signals from the $\mu$-PIC are amplified and discriminated by the preamplifier cards attached to the rear of the mother board. The preamplifier cards output both analog and digital signals. The digital signals are encoded by the position encoding module which consists of five field programmable gate arrays (FPGAs) and recorded by the memory module which works at the clock of $20 \mathrm{MHz}$. Analog signals from the 16 cathode strips are summed and digitized by the $100 \mathrm{MHz}$ flash ADC (FADC:REPIC RPV-160) to determine the event energy.

In order to check the performance of the $\mu$-PIC and the readout system for high rate signals, we irradiated the $\mu$-PIC with the $\mathrm{X}$-rays from the $\mathrm{X}$-ray generator. The acceleration voltage was $20 \mathrm{kV}$ and the intensity was controlled by way of varying the tube current between 0.01 and $0.6 \mathrm{~mA}$. The data acquisition rate is plotted against the X-ray intensity in Fig. 8. We achieved the maximum data acquisition rate of $7.7 \mathrm{MHz}$. This rate is twice higher than our previous readout system [8]. The saturation is thought to be due to the software for the FPGAs and optimizations are now under way.

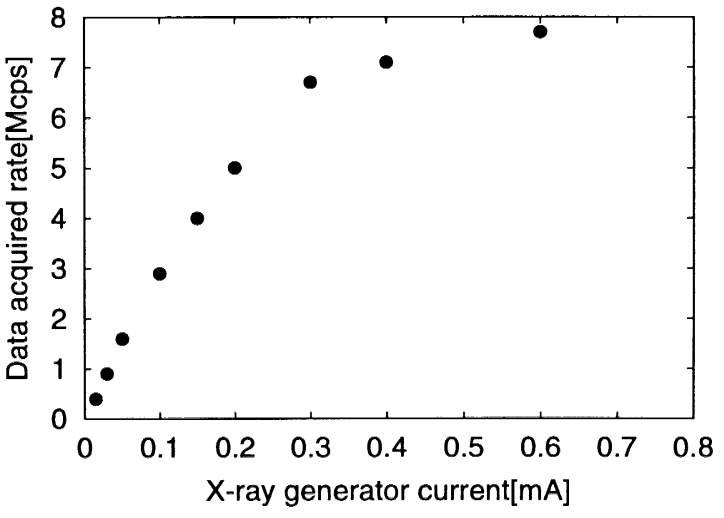

Fig. 8. Result of the high rate test of the $\mu$-PIC and the readout system.

\section{DESIGN OF THE MiCRO-TPC}

With the $\mu$-PIC and the readout electronics system described above, we developed a time projection chamber (TPC) which has fine time and spatial resolutions [7]. A gas enclosure with a drift length of $8 \mathrm{~cm}$ was attached to the $\mu$-PIC. Schematic drawings of the TPC gas enclosure is shown in Fig. 9. A negative high voltage of $3.3 \mathrm{kV}$ is supplied to the $1 \mathrm{~mm}$ thick aluminum window which is connected to the aluminum plane of $0.3 \mathrm{~mm}$ thick through $22 \mathrm{M} \Omega$. Fifteen field cage electrodes with $4 \mathrm{~mm}$ widths and $1 \mathrm{~mm}$ spacings are connected to the aluminum plane in series through $10 \mathrm{M} \Omega$ resistors. The electric field of $0.4 \mathrm{kV} / \mathrm{cm}$ is produced uniformly in the inner region.

\section{Performance of the Micro-TPC}

\section{A. Drift Velocity}

We measured the drift velocity of electrons and the gain dependence on the drift length in the micro-TPC using the cosmic-ray muons. We placed two plastic scintillators on both sides of the micro-TPC perpendicular to the direction of the electric field. We acquired the micro-TPC events in coincidence 


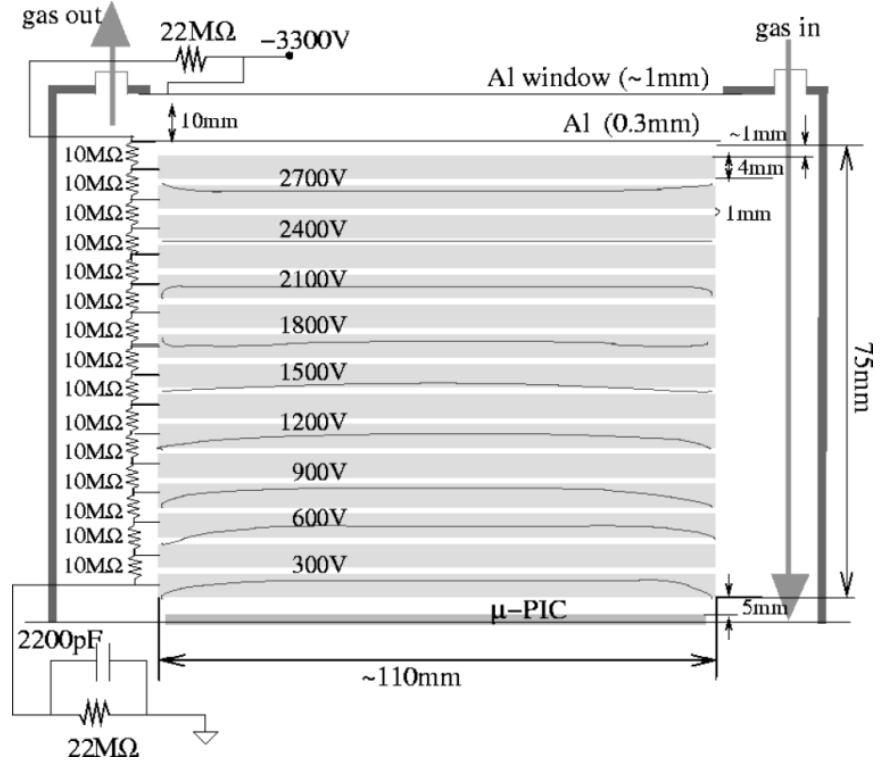

Fig. 9. Structure of the time projection chamber.

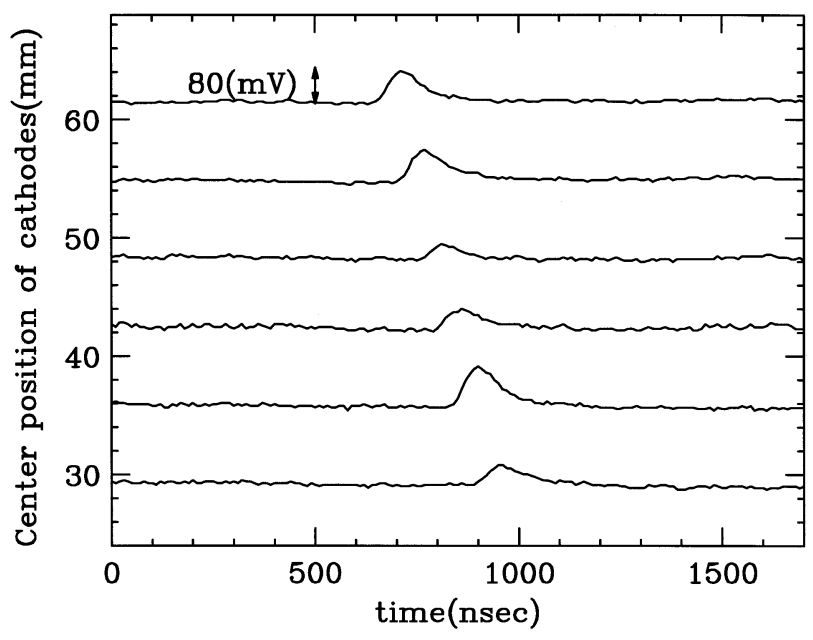

Fig. 10. A typical cosmic ray muon signal read from the cathode strips.

with both of the two scintillators. Fig. 10 shows the shapes of signals from cathode electrodes for a cosmic-ray muon. The signals are not identical because of the fluctuation of the gas gain due to the shape of the anode electrodes. The drift velocity was measured at various electric fields. The result shown in Fig. 11 is consistent with the previous measurement [9]. No gain difference was observed throughout the drift length.

\section{B. Three-Dimensional Trackings}

We detected the tracks of the electrons from a ${ }^{90} \mathrm{Sr} /{ }^{90} \mathrm{Y}$ radioactive source which emits electrons with the maximum kinetic energy of $0.546 \mathrm{MeV}$ and $2.282 \mathrm{MeV}$. The Micro-TPC was triggered by a plastic scintillator of $6 \times 6 \times 2 \mathrm{~mm}^{3}$ placed between the radioactive source and the micro-TPC. The $\mu$-PIC was operated at the gas gain of 7000. One of the typical 3-D tracks is shown in the upper panel of Fig. 12, while the projections of the several tracks on the $\mu$-PIC plane are shown in the lower.

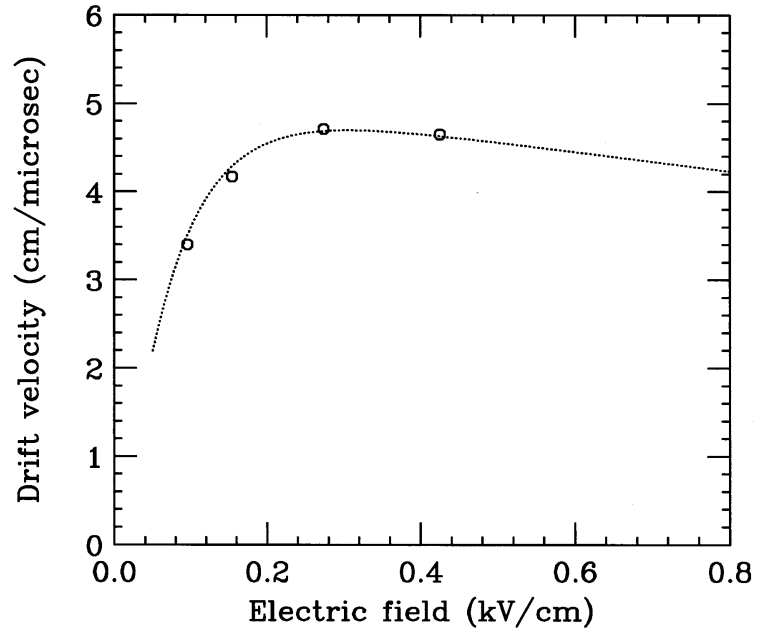

Fig. 11. Measured drift velocity of electrons as a function of the electric field (circle). The dotted line is the result of another group [9].

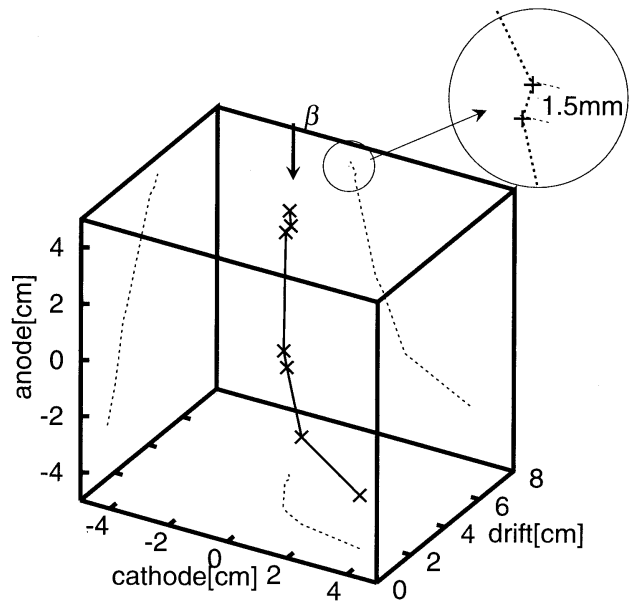

(a)

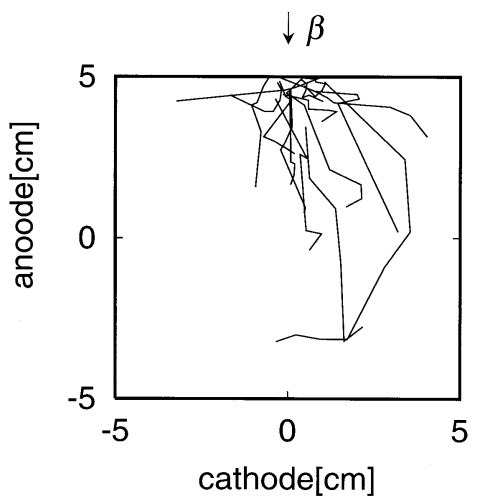

(b)

Fig. 12. (a) A typical 3-D track of an electron and (b) several tracks projected to the $\mu$-pic plane.

We only detected the points where large energy deposition took place because of the insufficient gas gain, therefore the electron tracks were detected as the dispersed points. Since the principle of the micro-TPC is confirmed, dense tracks like ones taken by a cloud chamber will be taken when we've achieved a sufficient gain. 


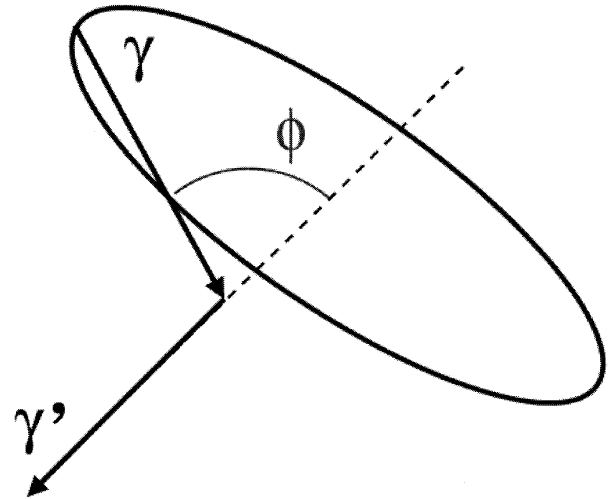

Fig. 13. Principle of the gamma-ray reconstruction by the double Compton method.

\section{Development of the Prototype MeV Gamma-Ray Imaging Detector}

One of the most interesting applications of the micro-TPC is a $\mathrm{MeV}$ gamma-ray imaging detector which makes an eventby-event reconstruction possible [10]. Typical MeV gammaray imaging detectors of these days with double or multiple Compton method [11], [12] measure the energy deposited to the electron $\left(E_{e}\right)$, the energy of the scattered gamma-ray $\left(E_{\gamma^{\prime}}\right)$ and the direction of the scattered gamma-ray $\left(\overrightarrow{\gamma^{\prime}}\right)$. The scattering angle of the gamma-ray $(\phi)$ is calculated by the following:

$$
\begin{aligned}
\cos \phi & =1-m_{e} c^{2}\left(\frac{1}{E_{\gamma^{\prime}}}-\frac{1}{E_{\gamma}}\right) \\
E_{\gamma} & =E_{\gamma^{\prime}}+E_{e} .
\end{aligned}
$$

Here, $m_{e}$ is the mass of the electron. The event cone is determined from $\overrightarrow{\gamma^{\prime}}$ and $\phi$. The principle of the gamma-ray reconstruction with double Comptom method is shown in Fig. 13.

Our new concept for the $\mathrm{MeV}$ gamma-ray imaging is to measure the direction of the Compton scattered electrons $(\vec{e})$ with the micro-TPC in addition to $E_{e}, E_{\gamma^{\prime}}$, and $\overrightarrow{\gamma^{\prime}}$. Since the micro-TPC can detect the track of the electrons as we have shown in Section IV, the hybrid detector of the micro-TPC and the enclosing scintillators can realize this new concept. With this new concept detector, we can determine another angle $\delta$ so that the incident gamma-ray is reconstructed for each event. The principle is shown in Fig. 14.

We have a redundant measured value $\alpha$ which can be used for the background rejection. Since no collimators are necessary, a large field of view can be realized.

We developed a prototype of the $\mathrm{MeV}$ gamma-ray imaging detector with the micro-TPC of $10 \times 10 \times 8 \mathrm{~cm}^{3}$ volume and an $\mathrm{NaI}(\mathrm{Tl})$ scintillator of $4^{\prime \prime} \times 4^{\prime \prime} \times 1^{\prime \prime}$ size read by 25 single anode PMTs of $3 / 4^{\prime \prime}$ diameters (Hamamatsu R116). The picture of the prototype is shown in Fig. 15.

We tested the prototype with a radioactive source of ${ }^{133} \mathrm{Ba}$ which mainly radiates $356 \mathrm{keV}$ gamma-rays. The setup is shown in Fig. 16. We set the origin of the coordinates at the center of the $\mu$-PIC detection plane and $X, Y$ and $Z$ axes are taken along the cathode, anode and drift direction of the micro-TPC, respectively. The radioactive source was set at $(-4.0,-4.3,-4.8)$ without any collimators and the center of the $\mathrm{NaI}$ (Tl) scintillator was set at $(-5.5,-5.5,22.9)$.

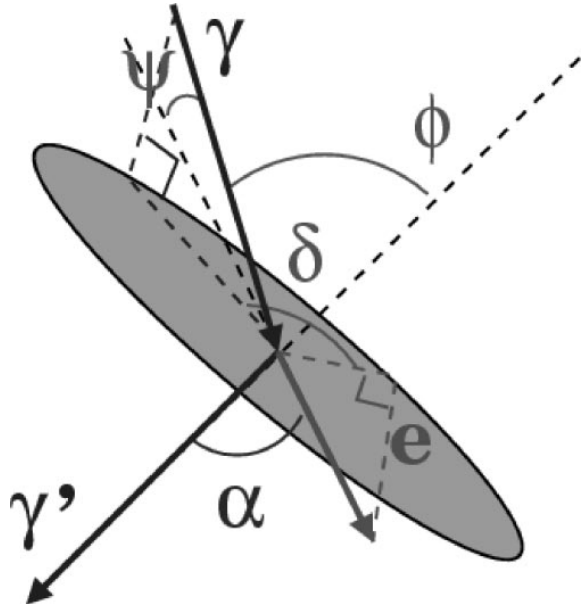

Fig. 14. Principle of the gamma-ray reconstruction by this new concept detector.

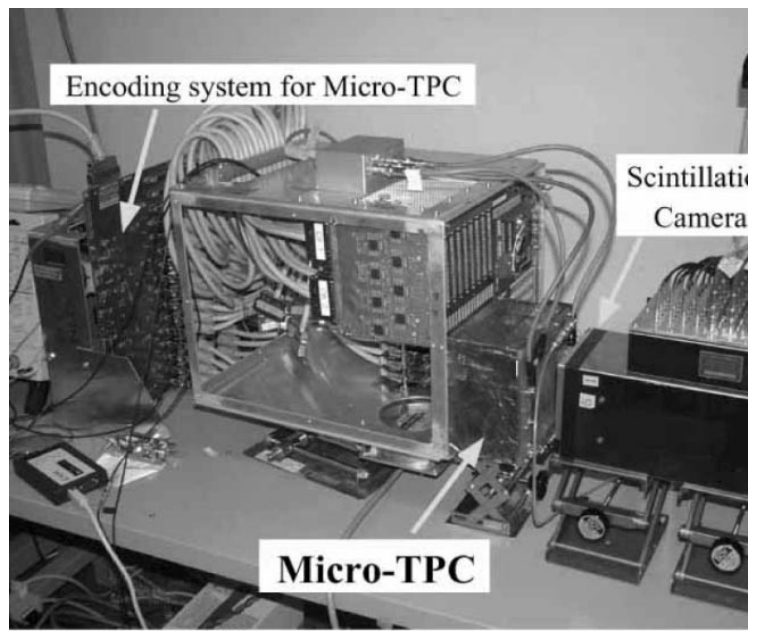

Fig. 15. The prototype of the MeV gamma-ray imaging detector.

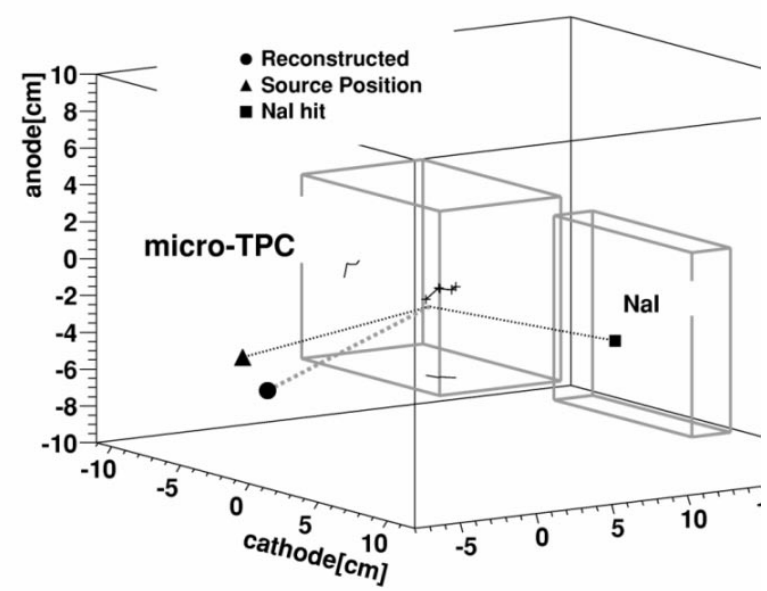

Fig. 16. One of the well-reconstructed gamma-rays taken by the prototype $\mathrm{MeV}$ gamma-ray detector.

Electron tracks are detected by the micro-TPC, while the energy and the position of the gamma-rays are taken by the $\mathrm{NaI}$ (Tl) scintillator. A gate of two- $\mu$ s was opened after the $\mathrm{NaI}(\mathrm{Tl})$ 
trigger for the micro-TPC signals and coincident events were recorded. We operated the $\mu$-PIC with a gas gain of 5000. For this concept test, we did not use the information on the electron energy but assumed $E_{e}=E_{\gamma}-E_{\gamma^{\prime}}$ as we knew the source energy. This test has much meaning since the $\mathrm{MeV}$ gamma-ray imaging for the radioactive sources of a know energy is useful for the medical use.

One of the well-reconstructed events is shown in Fig. 16. The source is shown by the closed triangle. The electron track is shown in the solid line and its projections are also drawn by the thin solid lines. The measured energy by the $\mathrm{NaI}(\mathrm{Tl})$ scintillator was $260 \mathrm{keV}$ and the calculated position of the gamma-ray in the $\mathrm{NaI}(\mathrm{Tl})$ scintillator is shown by the closed square. We reconstructed the direction of the incident photon from $E_{\gamma}, E_{\gamma^{\prime}}, \overrightarrow{\gamma^{\prime}}$ and $\vec{e}$. The reconstructed point in the $Z=-4.8$ plane is $(-2.2$, $-5.7,-4.8)$ and is shown by the filled circle. The reconstruction was realized by the angular accuracy of $10^{\circ}$ for this event. The gamma-ray is well-reconstructed in this event so that we can claim that this new concept is a very promising method for the MeV gamma-ray imaging.

We are going to use the energy information of the electrons, so that we can realize the $\mathrm{MeV}$ gamma-ray imaging for the gamma-rays of unknown energies, or we can use the energy for the background rejection. Statistical studies are also under way and the angular resolution and the detection efficiency will appear in another report.

\section{CONCLUSION}

We improved the $\mu$-PIC with the detection area of $10 \times 10$ $\mathrm{cm}^{2}$ and obtained the maximum gas gain of more than $10^{4}$ without any other multipliers. We detected X-rays of the rate as high as $7.7 \mathrm{MHz}$ with the $\mu$-PIC and the pipeline readout electronics. We developed a micro-TPC with the $\mu$-PIC readout and took 3-D electron tracks. We developed a prototype of the
$\mathrm{MeV}$ gamma-ray imaging detector with the micro-TPC and an $\mathrm{NaI}(\mathrm{Tl})$ scintillator so that we showed that this is a promising detector for the MeV gamma-ray imaging.

\section{REFERENCES}

[1] A. Oed, "Position-sensitive detector with microstrip anode for electron multiplication with gases," Nucl. Instrum. Methods, vol. A263, no. 2-3, pp. 351-359, Jan. 1988.

[2] T. Tanimori, A. Ochi, S. Minami, and T. Nagae, "Development of an imaging microstrip gas chamber with a $5 \times 5 \mathrm{~cm}^{2}$ area based on multi-chip module technology," Nucl. Instrum. Methods, vol. A381, no. 2-3, pp. 280-288, Nov. 1996.

[3] S. F. Biagi and T. J. Jones, "The microdot gas avalanche chamber: An investigation of new geometries," Nucl. Instrum. Methods, vol. A361, no. 1-2, pp. 72-76, July 1995.

[4] A. Ochi, T. Nagayoshi, S. Koishi, T. Tanimori, T. Nagae, and M. Nakamura, "A new design of the gaseous imaging detector: Micro pixel chamber," Nucl. Instrum. Methods, vol. A471, no. 1-2, pp. 264-267, Sept. 2001.

[5] - "Development of micro pixel chamber," Nucl. Instrum. Methods, vol. A478, no. 1-2, pp. 196-199, Feb. 2002.

[6] T. Nagayoshi, H. Kubo, K. Miuchi, A. Ochi, R. Orito, A. Takada, T. Tanimori, and M. Ueno, "Performance of large area micro pixel chamber," Nucl. Instrum. Methods, to be published.

[7] H. Kubo, K. Miuchi, T. Nagayoshi, A. Ochi, R. Orito, A. Takada, T. Tanimori, and M. Ueno, "Development of the time projection chamber with micro pixel electrodes," Nucl. Instrum. Methods, to be published.

[8] T. Tanimori, Y. Nishi, A. Ochi, and Y. Nishi, "Imaging gaseous detector based on micro-processing technology," Nucl. Instrum. Methods, vol. A436, no. 1-2, pp. 188-195, October 1999.

[9] U. Becker, R. Dinner, E. Fortunato, J. Kirchner, K. Rosera, A. Takada, and Y. Uchida, "Consistent measurements comparing the drift features of noble gas mixtures," Nucl. Instrum. Methods, vol. A421, no. 1-2, pp. 54-59, Jan. 1999.

[10] R. Orito, T. Tanimori, H. Kubo, K. Miuchi, T. Nagayoshi, A. Takada, and $\mathrm{M}$. Ueno, "A novel design of the MeV gamma-ray imaging detector with Micro-TPC," Nucl. Instrum. Methods, to be published.

[11] V. Schönfelder, "The imaging gamma-ray telescope COMPTEL aboard GRO," Adv. Space Res., vol. 11, no. 8, pp. 313-322, 1991.

[12] P. F. Bloser, R. Andritschke, G. Kanbach, V. Schönfelder, F. Schopper, and A. Zoglauer, "The MEGA advanced compton telescope project," New Astron. Rev., vol. 46, no. 8-10, pp. 611-616, July 1992. 